\title{
Taxonomy of the mycobiota of phytopatogenic fungi of Uzbekistan
}

\author{
Shukhrat Kamilov ${ }^{*}$, Mukhiddin Mamiev ${ }^{1}$, Samad Utaganov ${ }^{1}$, and Lola Babajanova ${ }^{1}$ \\ ${ }^{1}$ Tashkent State Agrarian University, 100140 Tashkent province, Uzbekistan
}

\begin{abstract}
This article provides data on the modern taxonomy of the mycobiota of phytopathogenic fungi (with the exception of Coelomycetes) identified in Uzbekistan. This article is based on the published data of previous generations of phytopathologists and mycologists in Uzbekistan and adjacent territories, as well as colleagues and their own research. Currently, the mycobiota of Uzbekistan includes 816 species from 122 genera, 112 families and 39 orders.
\end{abstract}

\section{Introduction}

The importance of classical works in biology as a general science is beyond doubt. Biology, like other general sciences, includes a large conglomerate of knowledge, which is divided into separate branches, namely separate sciences, including the classical ones botany, zoology, microbiology and others, as well as the presence of modern ones $[1,2]$.

The significance of the work on the classification of living organisms originates from the work of Linnaeus, Lamarck and a large number of other scientists [3].

It is now determined that the world of living organisms is different. There are animals, plants, fungi, microorganisms, actinomycetes and viruses (including mycoplasmas) [4].

A large number of living organisms are known on the globe. It is believed that only phytopathogenic fungi, there are more than 100,000 species. But to the end, we do not fully know the presence of living organisms, including fungi, of species in biological definition $[5,6]$.

This entire complex is characterized by extreme diversity, but all organisms have their own specific phylogenetic history and are related to each other by a certain degree of kinship. The main task of taxonomy is to identify connections between individual groups and combine them into various systematic categories [7].

However, according to the opinion of the classics of biology, for mushrooms, including, according to BK Kalymbetov [1], nature does not represent something frozen, finally formed and unchanged.

It should be noted, according to A.A. Yachevsky [1], the study of the flora of fungi of individual regions, districts or territories is in itself the primary task of mycologists, i.e. floristic research in the field of mycology is the first step, absolutely necessary for all further researchers, allowing to pose various problems and outline ways to solve them.

* Corresponding author: agrobiotechinfo@yandex.ru 
Phytopathogenic micromycetes - pathogens of plant diseases that cause damage to agriculture and ornamental economy, are of natural interest to mycologists and phytopathologists [8].

In this connection, the task of this work is to convey information about the knowledge and modern taxonomy of mycobiota, basically, phytopathogenic micromycetes of Uzbekistan, northwestern part of Central Asia [1,9].

This article is based on the published data of previous generations of phytopathologists and mycologists in Uzbekistan and adjacent territories, as well as colleagues and their own research [10].

\section{Materials and methods}

Considering the formation of phytopathogenic mycobiota, it should be noted that its composition arises as a result of complex relationships between fungi, plants and the environment and represents a non-random set of species, but a completely natural selection of them, which is formed under the influence of general physical-geographical and historical conditions of the area, as well as bio-ecological features [4].

The history of the development of phytopathological and mycological research in Uzbekistan and adjacent territories is associated with the work of NG Zaprometov, PN Golovin, TS Panfilova, NI Gaponenko, SS Ramazanova, BA Khasanov and many other researchers [5-8].

The purpose of this article was to provide data on the modern taxonomy of the mycobiota of Uzbekistan, based on the phytopathogenic composition, according to the modern taxonomy of fungi, not excluding the classical taxonomy of Saccardo, according to the works of Kirk and others [6] and Mclaughin and Spatofora [5].

\section{Results and discussion}

This article presents the results of data on the composition of fungi - micromycetes noted in the conditions of Uzbekistan. Table 1 shows the data of the modern taxonomic position of micromycete fungi (with the exception of the group of orders of melanconial and pycnidial fungi - Coelomycetes).

Currently, the mycobiota of Uzbekistan includes 816 species from 122 genera, 112 families and 39 orders (Table 1).

At present, representatives of fungi are noted in the composition of micromycete fungi, and according to modern taxonomy, fungal-like organisms (Table 1).

According to the classical system Saccardo, oomycetes and chytridiomycetes belong to the lowest fungi. In this connection, we, the representatives of Oomycota, are discussing in this article. According to modern views, oomycetes are fungi-like organisms.

The oomycetes include 132 species belonging to 6 genera, 2 families, and 9 orders. Saprotropic fungi are widespread in water bodies as saprotrophs on plant debris and animal substrates. Their harmfulness is known in fish breeding. This group also includes species of the Pythiaceae families - typical soil saprotrophs and causative agents of root rot of cultivated plants, Albuginaceae and Peronosporaceae - parasites of higher plants. Due to their species richness and practical importance, mushrooms from the genera Pithium and Phytophthora are of great interest. 
Table 1. Composition and taxonomy of mycobiota of Uzbekistan.

\begin{tabular}{|c|c|c|c|c|c|c|c|c|}
\hline Phylum & Class & 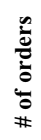 & Order & 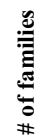 & Families & 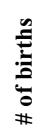 & Species & 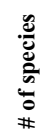 \\
\hline \multirow{18}{*}{$\underset{*}{\text { Oomycota }}$} & \multirow{18}{*}{$\begin{array}{l}\text { Oomyce } \\
\text { tes }\end{array}$} & \multirow{18}{*}{9} & \multirow{7}{*}{$\begin{array}{l}\text { Saprolegni } \\
\text { ales }\end{array}$} & \multirow{7}{*}{2} & \multirow{6}{*}{$\begin{array}{l}\text { Saprolegni } \\
\text { aceae }\end{array}$} & \multirow{6}{*}{6} & $\begin{array}{c}\text { Aphanomyce } \\
s \mathrm{DB}\end{array}$ & 4 \\
\hline & & & & & & & Achlya Nees & 9 \\
\hline & & & & & & & $\begin{array}{l}\text { Dictyushus } \\
\text { Leitg. }\end{array}$ & 2 \\
\hline & & & & & & & $\begin{array}{l}\text { Saprolegnia } \\
\text { Nees }\end{array}$ & 8 \\
\hline & & & & & & & $\begin{array}{c}\text { Protoachlya } \\
\text { Coker }\end{array}$ & 1 \\
\hline & & & & & & & $\begin{array}{l}\text { Isoachlya } \\
\text { Kauffman }\end{array}$ & 3 \\
\hline & & & & & $\begin{array}{l}\text { Leptolegni } \\
\text { ellaceae }\end{array}$ & 1 & $\begin{array}{l}\text { Aphanomyco } \\
\text { psis Scherff. }\end{array}$ & 1 \\
\hline & & & \multirow{2}{*}{$\begin{array}{c}\text { Leptomital } \\
\text { es }\end{array}$} & \multirow{2}{*}{1} & \multirow{2}{*}{$\begin{array}{l}\text { Leptomita } \\
\text { ceae }\end{array}$} & \multirow{2}{*}{2} & $\begin{array}{l}\text { Leptomitus } \\
\text { C.Agardh }\end{array}$ & 1 \\
\hline & & & & & & & $\begin{array}{l}\text { Apodachlya } \\
\text { Pringsh. }\end{array}$ & 1 \\
\hline & & & \multirow{2}{*}{$\begin{array}{l}\text { Rhipidiale } \\
\text { s }\end{array}$} & \multirow{2}{*}{1} & \multirow{2}{*}{$\begin{array}{c}\text { Rhipidiace } \\
\text { ae }\end{array}$} & \multirow{2}{*}{2} & $\begin{array}{l}\text { Rhipidium } \\
\text { Cornu }\end{array}$ & 1 \\
\hline & & & & & & & $\begin{array}{l}\text { Sapromyces } \\
\text { Fritsch }\end{array}$ & 1 \\
\hline & & & $\begin{array}{l}\text { Albuginale } \\
\mathrm{s}\end{array}$ & 1 & $\begin{array}{l}\text { Albuginac } \\
\text { eae }\end{array}$ & 1 & $\begin{array}{c}\text { Albugo } \\
\text { (Pers.) } \\
\text { Roussel }\end{array}$ & 3 \\
\hline & & & \multirow{2}{*}{ Pythiales } & \multirow{2}{*}{2} & Pythiaceae & 1 & $\begin{array}{l}\text { Pythium } \\
\text { Pringsh. }\end{array}$ & 3 \\
\hline & & & & & $\begin{array}{l}\text { Pytiogeton } \\
\text { aceae }\end{array}$ & 1 & $\begin{array}{l}\text { Pytiogeton } \\
\text { Minden }\end{array}$ & 1 \\
\hline & & & \multirow{4}{*}{$\begin{array}{l}\text { Peronospo } \\
\text { rales }\end{array}$} & \multirow{4}{*}{1} & \multirow{4}{*}{$\begin{array}{l}\text { Peronospo } \\
\text { raceae }\end{array}$} & \multirow{4}{*}{4} & $\begin{array}{c}\text { Sclerospora } \\
\text { J.Shrot }\end{array}$ & 1 \\
\hline & & & & & & & $\begin{array}{l}\text { Peronospora } \\
\text { Corda }\end{array}$ & 75 \\
\hline & & & & & & & $\begin{array}{c}\text { Phytophthora } \\
\text { DB. }\end{array}$ & 1 \\
\hline & & & & & & & $\begin{array}{l}\text { Plasmopara } \\
\text { J.Schrot. }\end{array}$ & 4 \\
\hline \multirow{4}{*}{$\begin{array}{l}\text { Chytridio } \\
\text { mycota }\end{array}$} & \multirow{4}{*}{$\begin{array}{l}\text { Chytridi } \\
\text { omycete } \\
\text { s }\end{array}$} & \multirow{4}{*}{3} & $\begin{array}{l}\text { Olpidiopsi } \\
\text { dales }\end{array}$ & 1 & $\begin{array}{l}\text { Olpidiopsi } \\
\text { daceae }\end{array}$ & 1 & $\begin{array}{l}\text { Olpidiopsis } \\
\text { Cornu }\end{array}$ & 1 \\
\hline & & & \multirow{2}{*}{$\begin{array}{l}\text { Spizellom } \\
\text { ycetales }\end{array}$} & \multirow[t]{2}{*}{2} & $\begin{array}{c}\text { Olpidiacea } \\
\mathrm{e}\end{array}$ & 1 & $\begin{array}{c}\text { Olpidium } \\
\text { (A.Braun) } \\
\text { J.Schrot. }\end{array}$ & 5 \\
\hline & & & & & $\begin{array}{l}\text { Spizellom } \\
\text { ycetaceae }\end{array}$ & 1 & $\begin{array}{c}\text { Rhizophlyctis } \\
\text { A.Fisch. }\end{array}$ & 1 \\
\hline & & & $\begin{array}{c}\text { Chytridiale } \\
\mathrm{s}\end{array}$ & 1 & $\begin{array}{c}\text { Chytridiac } \\
\text { eae }\end{array}$ & 1 & $\begin{array}{l}\text { Sporophlyctis } \\
\text { Serbinow }\end{array}$ & 1 \\
\hline \multirow{2}{*}{$\begin{array}{c}\text { Monoblep } \\
\text { haridimyco } \\
\text { ta }\end{array}$} & \multirow{2}{*}{$\begin{array}{l}\text { Monobl } \\
\text { epharidi } \\
\text { mycetes }\end{array}$} & \multirow{2}{*}{1} & \multirow{2}{*}{$\begin{array}{l}\text { Monoblep } \\
\text { haridales }\end{array}$} & \multirow{2}{*}{2} & $\begin{array}{l}\text { Monoblep } \\
\text { haridaceae }\end{array}$ & 1 & $\begin{array}{c}\text { Monoblephar } \\
\text { ales Cornu }\end{array}$ & 1 \\
\hline & & & & & $\begin{array}{l}\text { Harpochyt } \\
\text { riaceae }\end{array}$ & 1 & $\begin{array}{l}\text { Harpochytriu } \\
m \text { Laqgerh. }\end{array}$ & 1 \\
\hline Blastocladi & Blastocl & 1 & Blastocladi & 1 & Blastocladi & ? & $\begin{array}{l}\text { Allomyces } \\
\text { E.J.Butler }\end{array}$ & 1 \\
\hline omycota & $\begin{array}{l}\text { adiomyc } \\
\text { etes }\end{array}$ & 1 & ales & 1 & aceae & 2 & $\begin{array}{l}\text { Blastocladia } \\
\text { Reinsch }\end{array}$ & 1 \\
\hline
\end{tabular}




\begin{tabular}{|c|c|c|c|c|c|c|c|c|}
\hline \multirow{10}{*}{$\begin{array}{c}\text { Zygomyco } \\
\text { ta }\end{array}$} & \multirow{10}{*}{$\begin{array}{c}\text { Mucoro } \\
\text { mycotin } \\
\mathrm{a}\end{array}$} & \multirow{10}{*}{2} & $\begin{array}{l}\text { Mortierella } \\
\text { les }\end{array}$ & 1 & $\begin{array}{l}\text { Mortierella } \\
\text { ceae }\end{array}$ & 2 & $\begin{array}{l}\text { Mortierella } \\
\text { Coem. }\end{array}$ & 2 \\
\hline & & & \multirow{9}{*}{ Mucorales } & \multirow{9}{*}{3} & \multirow{7}{*}{$\begin{array}{c}\text { Mucoracea } \\
\mathrm{e}\end{array}$} & \multirow{7}{*}{7} & $\begin{array}{l}\text { Helicostylum } \\
\text { Corda }\end{array}$ & 1 \\
\hline & & & & & & & $\begin{array}{l}\text { Thamnidium } \\
\text { Lk. }\end{array}$ & 1 \\
\hline & & & & & & & $\begin{array}{c}\text { Absidia } \\
\text { Tiegh. }\end{array}$ & 1 \\
\hline & & & & & & & $\begin{array}{l}\text { Actinomucor } \\
\text { Schostak. }\end{array}$ & 1 \\
\hline & & & & & & & $\begin{array}{c}\text { Rhizopus } \\
\text { Exrenb. }\end{array}$ & 3 \\
\hline & & & & & & & $\begin{array}{c}\text { Circinella } \\
\text { Tiegh.\& } \\
\text { G.Le Monn. }\end{array}$ & 2 \\
\hline & & & & & & & $\begin{array}{c}\text { Mucor } \\
\text { P.Micheli ex } \\
\text { Fr. } \\
\end{array}$ & 12 \\
\hline & & & & & $\begin{array}{l}\text { Cunningha } \\
\text { mellaceae }\end{array}$ & 1 & $\begin{array}{l}\text { Cunningham } \\
\text { ella Matr. }\end{array}$ & 1 \\
\hline & & & & & $\begin{array}{l}\text { Syncephal } \\
\text { astraceae }\end{array}$ & 1 & $\begin{array}{c}\text { Syncephalast } \\
\text { rum } \\
\text { J.Schroet. }\end{array}$ & 1 \\
\hline \multirow{20}{*}{$\begin{array}{c}\text { Ascomycot } \\
\text { a }\end{array}$} & $\begin{array}{c}\text { Taphrin } \\
\text { omycete } \\
\mathrm{s} \\
\end{array}$ & 1 & $\begin{array}{c}\text { Taphrinale } \\
\text { s }\end{array}$ & 1 & $\begin{array}{c}\text { Taphrinace } \\
\text { ae }\end{array}$ & 1 & Taphrina Fr. & 4 \\
\hline & \multirow{12}{*}{$\begin{array}{l}\text { Leotiom } \\
\text { ycetes }\end{array}$} & \multirow{12}{*}{1} & \multirow{12}{*}{$\begin{array}{c}\text { Erysiphale } \\
\text { s }\end{array}$} & \multirow{12}{*}{1} & \multirow{12}{*}{$\begin{array}{c}\text { Erysiphace } \\
\text { ae }\end{array}$} & \multirow{12}{*}{12} & $\begin{array}{c}\text { Erysiphe } \\
\text { R.Hedw. ex } \\
\text { DC. }\end{array}$ & 16 \\
\hline & & & & & & & $\begin{array}{c}\text { Microsphaer } \\
a \text { Lev. }\end{array}$ & 13 \\
\hline & & & & & & & $\begin{array}{c}\text { Blumeria } \\
\text { Golovin ex } \\
\text { Speer } \\
\end{array}$ & 1 \\
\hline & & & & & & & $\begin{array}{l}\text { Arthrocladiel } \\
\text { la Vassilkov }\end{array}$ & 1 \\
\hline & & & & & & & $\begin{array}{l}\text { Golovinomyc } \\
\text { es (U.Braun) } \\
\text { V.P.Gelyuta }\end{array}$ & 12 \\
\hline & & & & & & & $\begin{array}{l}\text { Podosphaera } \\
\text { Kunze }\end{array}$ & 3 \\
\hline & & & & & & & $\begin{array}{c}\text { Sphaerotheca } \\
\text { Lev. }\end{array}$ & 18 \\
\hline & & & & & & & $\begin{array}{c}\text { Phyllactinia } \\
\text { Lev. }\end{array}$ & 7 \\
\hline & & & & & & & $\begin{array}{l}\text { Leveillula } \\
\text { G.Arnaud } \\
\end{array}$ & 9 \\
\hline & & & & & & & $\begin{array}{l}\text { Sawadeae } \\
\text { Miyabe }\end{array}$ & 2 \\
\hline & & & & & & & $\begin{array}{c}\text { Uncinula } \\
\text { Lev. }\end{array}$ & 5 \\
\hline & & & & & & & $\begin{array}{l}\text { Uncinuliella } \\
\text { R.Y.Sheng \& } \\
\text { G.O.Chen } \\
\end{array}$ & 1 \\
\hline & \multirow{6}{*}{$\begin{array}{l}\text { Eurotim } \\
\text { ycetes }\end{array}$} & \multirow{6}{*}{2} & \multirow{3}{*}{$\begin{array}{l}\text { Onygenale } \\
\mathrm{s}\end{array}$} & \multirow{3}{*}{2} & \multirow{2}{*}{$\begin{array}{l}\text { Gymnoasc } \\
\text { aceae }\end{array}$} & \multirow{2}{*}{2} & $\begin{array}{c}\text { Gymnoascus } \\
\text { Baran. }\end{array}$ & 2 \\
\hline & & & & & & & $\begin{array}{l}\text { Arachniotus } \\
\text { J.Schroet. }\end{array}$ & 1 \\
\hline & & & & & $\begin{array}{c}\text { Onygenace } \\
\text { ae }\end{array}$ & 1 & $\begin{array}{c}\text { Amauroascus } \\
\text { J.Schroet. }\end{array}$ & 1 \\
\hline & & & \multirow{3}{*}{ Eurotiales } & \multirow{3}{*}{2} & $\begin{array}{c}\text { Thermoasc } \\
\text { aceae }\end{array}$ & 1 & $\begin{array}{c}\text { Thermoascus } \\
\text { Miehe }\end{array}$ & 1 \\
\hline & & & & & \multirow{2}{*}{$\begin{array}{c}\text { Trichocom } \\
\text { aceae }\end{array}$} & \multirow{2}{*}{2} & $\begin{array}{c}\text { Eurotium } \\
\text { Link } \\
\end{array}$ & 1 \\
\hline & & & & & & & $\begin{array}{c}\text { Emericella } \\
\text { Berk. } \\
\end{array}$ & 1 \\
\hline & $\begin{array}{l}\text { Sordario } \\
\text { mycetes }\end{array}$ & 9 & $\begin{array}{l}\text { Phyllachor } \\
\text { ales }\end{array}$ & 1 & $\begin{array}{l}\text { Phyllachor } \\
\text { aceae }\end{array}$ & 2 & $\begin{array}{l}\text { Polystigma } \\
\text { DC. }\end{array}$ & 3 \\
\hline
\end{tabular}




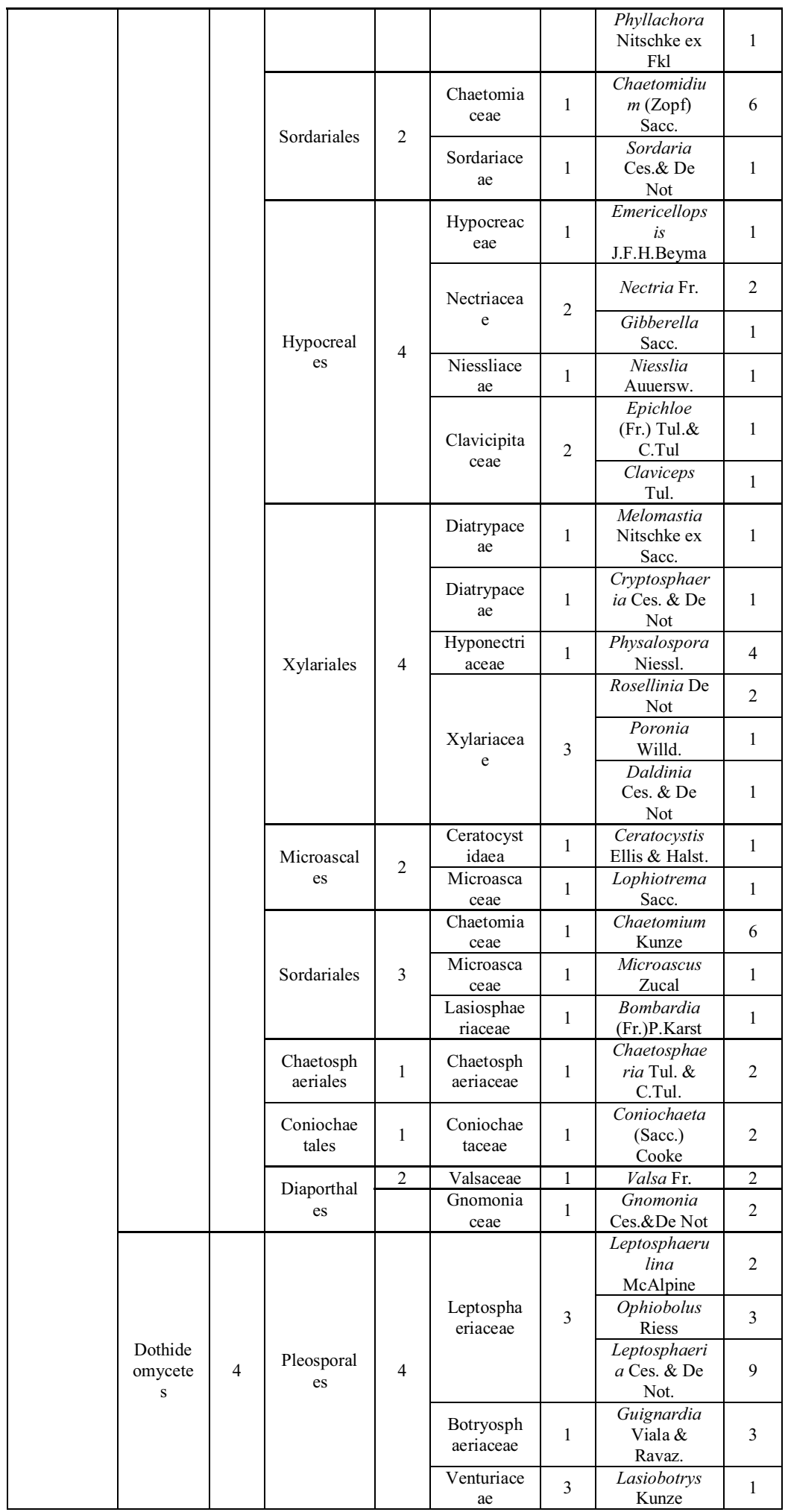




\begin{tabular}{|c|c|c|c|c|c|c|c|c|}
\hline & & & & & & & $\begin{array}{l}\text { Platychora } \\
\text { Petr. }\end{array}$ & 1 \\
\hline & & & & & & & $\begin{array}{l}\text { Venturia } \\
\text { Sacc. }\end{array}$ & 2 \\
\hline & & & & & $\begin{array}{l}\text { Didymosp } \\
\text { haeriaceae }\end{array}$ & 1 & $\begin{array}{c}\text { Didymosphae } \\
\text { ria Fuckel }\end{array}$ & \\
\hline & & & & & $\begin{array}{c}\text { Pleosporac } \\
\text { eae }\end{array}$ & & $\begin{array}{c}\text { Pyrenophora } \\
\text { Fr. }\end{array}$ & 4 \\
\hline & & & $\begin{array}{l}\text { Pleosporal } \\
\text { es }\end{array}$ & 1 & & 2 & $\begin{array}{c}\text { Pleospora } \\
\text { Rabenh. Ex } \\
\text { Ces. \& De } \\
\text { Not }\end{array}$ & 38 \\
\hline & & & & & Mycospha & 2 & $\begin{array}{l}\text { Mycosphaere } \\
\text { lla Johanson }\end{array}$ & 35 \\
\hline & & & $\begin{array}{c}\text { Capnodial } \\
\text { es }\end{array}$ & 2 & erellaceae & 2 & $\begin{array}{l}\text { Sphaerulina } \\
\text { Sacc. }\end{array}$ & 1 \\
\hline & & & & & $\begin{array}{l}\text { Capnodiac } \\
\text { eae }\end{array}$ & 1 & $\begin{array}{l}\text { Capnodium } \\
\text { Mont. }\end{array}$ & 2 \\
\hline & & & & & $\begin{array}{l}\text { Microthyri } \\
\text { aceae }\end{array}$ & 1 & $\begin{array}{l}\text { Microthyriu } \\
m \text { Desm. }\end{array}$ & 2 \\
\hline & & & & & $\begin{array}{c}\text { Phaeospha } \\
\text { riaceae }\end{array}$ & 1 & $\begin{array}{l}\text { Phaeosphari } \\
a \text { I.Miyake }\end{array}$ & 4 \\
\hline & & & & & Pleomassa & 2 & $\begin{array}{l}\text { Nodulosphae } \\
\text { ria Rabenh. }\end{array}$ & 2 \\
\hline & & & Microthyri & 6 & riaceae & 2 & $\begin{array}{c}\text { Trematospha } \\
\text { eria } \text { Fuckel }\end{array}$ & 4 \\
\hline & & & & & $\begin{array}{l}\text { Melanom } \\
\text { mataceae }\end{array}$ & 1 & $\begin{array}{c}\text { Melanomma } \\
\text { Nitschke ex } \\
\text { Fuckel }\end{array}$ & 2 \\
\hline & & & & & $\begin{array}{c}\text { Diademace } \\
\text { ae }\end{array}$ & 1 & $\begin{array}{l}\text { Comoclathris } \\
\text { Clem. }\end{array}$ & 2 \\
\hline & & & & & $\begin{array}{c}\text { Cucurbitar } \\
\text { iaceae }\end{array}$ & 2 & $\begin{array}{c}\text { Cucurbitaria } \\
\text { Gray }\end{array}$ & 2 \\
\hline & & & & & $\begin{array}{c}\text { Sarcoscyp } \\
\text { haceae }\end{array}$ & 1 & $\begin{array}{c}\text { Strickeria } \\
\text { Koerd. }\end{array}$ & 14 \\
\hline & & & & & $\begin{array}{c}\text { Sarcoscyp } \\
\text { haceae }\end{array}$ & 1 & $\begin{array}{l}\text { Sarcoscypha } \\
\text { (Fr.)Boud. }\end{array}$ & 1 \\
\hline & & & & & Morchella & 2 & $\begin{array}{c}\text { Morchella } \\
\text { Dill. ex Pers. }\end{array}$ & 3 \\
\hline & Pezizom & 1 & Pezizales & 5 & & & Verpa Sw. & 1 \\
\hline & & & & & $\begin{array}{c}\text { Helvellace } \\
\text { ae }\end{array}$ & 1 & Helvella $\mathrm{L}$. & 1 \\
\hline & & & & & Pyronemat & 2 & $\begin{array}{c}\text { Humaria } \\
\text { Fuckel }\end{array}$ & 1 \\
\hline & & & & & aceae & & $\begin{array}{c}\text { Geopora } \\
\text { Harkn. }\end{array}$ & 1 \\
\hline & & & $\begin{array}{l}\text { Rhytismat } \\
\text { ales }\end{array}$ & 1 & $\begin{array}{l}\text { Rhytismat } \\
\text { aceae }\end{array}$ & 2 & Rhytisma Fr. & 1 \\
\hline & & & & & $\begin{array}{l}\text { Sclerotinia } \\
\text { ceae }\end{array}$ & 1 & $\begin{array}{l}\text { Mollisia (Fr.) } \\
\text { P.Kars. }\end{array}$ & 2 \\
\hline & $\begin{array}{c}\text { Leotiom } \\
\text { ycetes }\end{array}$ & 2 & Helotiales & 3 & Sclerotinia & 2 & $\begin{array}{c}\text { Sclerotinia } \\
\text { Fuckel }\end{array}$ & 1 \\
\hline & & & Helotiales & & ceae & & $\begin{array}{c}\text { Pyrenopeziza } \\
\text { Fuckel }\end{array}$ & 1 \\
\hline & & & & & $\begin{array}{c}\text { Dermatace } \\
\text { ae }\end{array}$ & 1 & $\begin{array}{c}\text { Pseudopeziza } \\
\text { Fuckel }\end{array}$ & 3 \\
\hline & & & & & Pucciniastr & 2 & $\begin{array}{c}\text { Hyalopsora } \\
\text { Magnus }\end{array}$ & 1 \\
\hline & & & & & aceae & 2 & $\begin{array}{l}\text { Pucciniastru } \\
m \text { G.H.Otth }\end{array}$ & 1 \\
\hline & & & & & $\begin{array}{c}\text { Melampso } \\
\text { raceae }\end{array}$ & 1 & $\begin{array}{l}\text { Melampsora } \\
\text { Castagne }\end{array}$ & 19 \\
\hline $\begin{array}{l}\text { Basidiomy } \\
\text { cota }\end{array}$ & $\begin{array}{l}\text { Puccinio } \\
\text { mycetes }\end{array}$ & 1 & $\begin{array}{c}\text { Pucciniale } \\
\text { s }\end{array}$ & 6 & $\begin{array}{l}\text { Coleospori } \\
\text { aceae }\end{array}$ & 1 & $\begin{array}{c}\text { Chrysomyxa } \\
\text { Unger }\end{array}$ & 1 \\
\hline & & & & & $\begin{array}{c}\text { Uropyxida } \\
\text { ceae }\end{array}$ & 1 & $\begin{array}{c}\text { Tranzschelia } \\
\text { Arthur/ }\end{array}$ & 1 \\
\hline & & & & & Phragmidi & 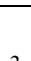 & $\begin{array}{l}\text { Phragmidium } \\
\text { Link }\end{array}$ & 11 \\
\hline & & & & & aceae & 3 & $\begin{array}{c}\text { Trachyspora } \\
\text { Fuckel }\end{array}$ & 1 \\
\hline
\end{tabular}




\begin{tabular}{|c|c|c|c|c|c|c|c|c|}
\hline & & & & & & & $\begin{array}{l}\text { Gymnoconia } \\
\text { Lagerh. }\end{array}$ & 1 \\
\hline & & & & & & & $\begin{array}{l}\text { Gymnospora } \\
\text { ngium } \\
\text { R.Hedw. ex } \\
\text { DC. }\end{array}$ & 4 \\
\hline & & & & & Pucciniace & 4 & $\begin{array}{c}\text { Uromyces } \\
\text { (Link)Unger }\end{array}$ & 56 \\
\hline & & & & & ae & 4 & $\begin{array}{c}\text { Puccinia } \\
\text { Pers. }\end{array}$ & 146 \\
\hline & & & & & & & $\begin{array}{c}\text { Miyagia } \\
\text { Miyabe ex } \\
\text { Syd. \& } \\
\text { P.Syd. } \\
\end{array}$ & 1 \\
\hline & & & & & Ustilagina & 2 & $\begin{array}{c}\text { Ustilago } \\
\text { (Pers.)Rousse } \\
1 .\end{array}$ & 45 \\
\hline & Ustilagi & 1 & Ustilaginal & 2 & & & $\begin{array}{c}\text { Tranzscheliel } \\
\text { la Lavrov }\end{array}$ & 1 \\
\hline & $\begin{array}{l}\text { es } \\
\text { es }\end{array}$ & 1 & es & 2 & & & $\begin{array}{c}\text { Anthracoidea } \\
\text { Bref. }\end{array}$ & 6 \\
\hline & & & & & $\begin{array}{c}\text { Anthracold } \\
\text { eaceae }\end{array}$ & 2 & $\begin{array}{c}\text { Tolyposporiu } \\
m \text { Woronin } \\
\text { ex J.Sroet. }\end{array}$ & 1 \\
\hline & $\begin{array}{l}\text { Microbo } \\
\text { mycetes }\end{array}$ & 1 & $\begin{array}{c}\text { Microbotr } \\
\text { yales }\end{array}$ & 1 & $\begin{array}{c}\text { Microbotr } \\
\text { yaceae }\end{array}$ & 1 & $\begin{array}{c}\text { Sphacelothec } \\
a \text { DB }\end{array}$ & 12 \\
\hline & Ustilagi & & & & $\begin{array}{c}\text { Glomospor } \\
\text { iaceae }\end{array}$ & 1 & $\begin{array}{l}\text { Thecaphora } \\
\text { Fingerh. }\end{array}$ & 1 \\
\hline & $\begin{array}{l}\text { nomycet } \\
\text { es }\end{array}$ & 1 & $\begin{array}{l}\text { les } \\
\text { locystida }\end{array}$ & 2 & $\begin{array}{l}\text { Urocystida } \\
\text { ceae }\end{array}$ & 1 & $\begin{array}{c}\text { Urocystis } \\
\text { Raben. ex } \\
\text { Fuckel } \\
\end{array}$ & 18 \\
\hline & Exobasi & 2 & Tilletiales & 1 & $\begin{array}{c}\text { Tilletiacea } \\
\mathrm{e}\end{array}$ & 1 & $\begin{array}{l}\text { Tilletia Tul. } \\
\text { \& C.Tul. }\end{array}$ & 11 \\
\hline & $\begin{array}{l}\text { tes } \\
\text { te }\end{array}$ & 2 & $\begin{array}{l}\text { Entylomat } \\
\text { ales }\end{array}$ & 1 & $\begin{array}{c}\text { Entylomat } \\
\text { aceae }\end{array}$ & 1 & $\begin{array}{l}\text { Entyloma } \\
\text { DB. }\end{array}$ & 16 \\
\hline & & & & & & & $\begin{array}{c}\text { Halobyssus } \\
\text { Zucal }\end{array}$ & 1 \\
\hline & & & & & & & $\begin{array}{l}\text { Monilia } \\
\text { Bonord. }\end{array}$ & 4 \\
\hline & & & & & & & $\begin{array}{c}\text { Oospora } \\
\text { Wallr. }\end{array}$ & 3 \\
\hline & & & & & & & $\begin{array}{l}\text { Geotrichum } \\
\text { Link }\end{array}$ & 1 \\
\hline & & & & & & & $\begin{array}{c}\text { Cephalospori } \\
\text { um Corda }\end{array}$ & 11 \\
\hline & & & & & & & $\begin{array}{l}\text { Acremonium } \\
\text { G.Arnaud ex } \\
\text { Cif. } \\
\end{array}$ & 2 \\
\hline & & & & & & & $\begin{array}{l}\text { Trichoderma } \\
\text { Pers. }\end{array}$ & 4 \\
\hline $\begin{array}{l}\text { Anamorphi } \\
\text { c fungi }\end{array}$ & Deutero & 1 & Hyphomec & 3 & Moniliacea & 52 & $\begin{array}{c}\text { Aspergillus } \\
\text { P.Micheli ex } \\
\text { Link }\end{array}$ & 33 \\
\hline ycota) & & & & & & & $\begin{array}{c}\text { Penicillium } \\
\text { Link }\end{array}$ & 70 \\
\hline & & & & & & & $\begin{array}{c}\text { Acrostalagm } \\
\text { us } \text { Corda }\end{array}$ & 1 \\
\hline & & & & & & & $\begin{array}{c}\text { Gliocladium } \\
\text { Corda }\end{array}$ & 8 \\
\hline & & & & & & & $\begin{array}{c}\text { Scopulariops } \\
\text { is Bainier }\end{array}$ & 3 \\
\hline & & & & & & & $\begin{array}{c}\text { Botrytis } \\
\text { P.Micheli ex } \\
\text { Pers. } \\
\end{array}$ & 3 \\
\hline & & & & & & & $\begin{array}{l}\text { Sporotrichum } \\
\text { Link } \\
\end{array}$ & 7 \\
\hline & & & & & & & $\begin{array}{c}\text { Spicaria } \\
\text { Harting } \\
\end{array}$ & 5 \\
\hline & & & & & & & $\begin{array}{l}\text { Verticillium } \\
\text { Nees }\end{array}$ & 14 \\
\hline
\end{tabular}




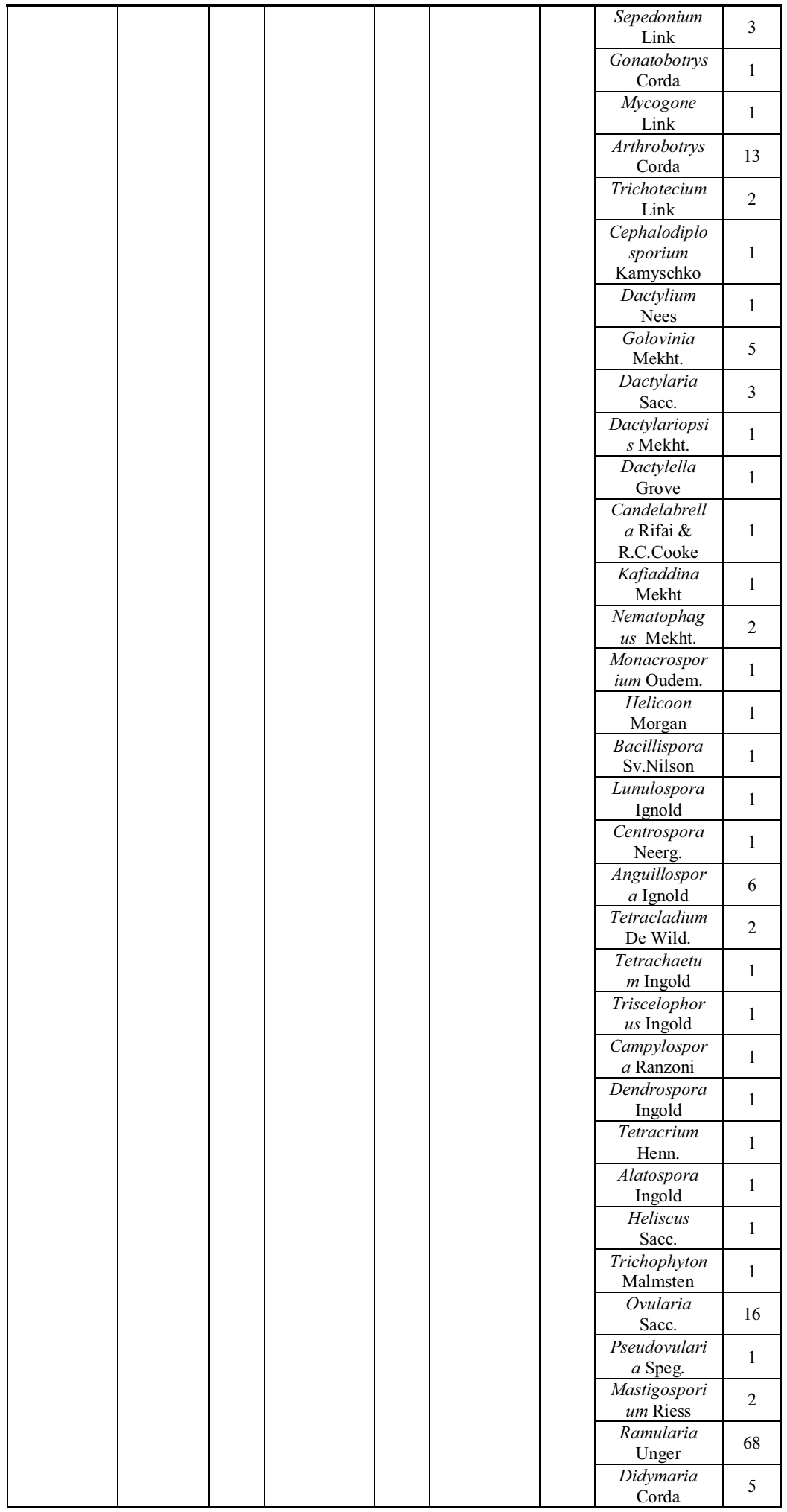




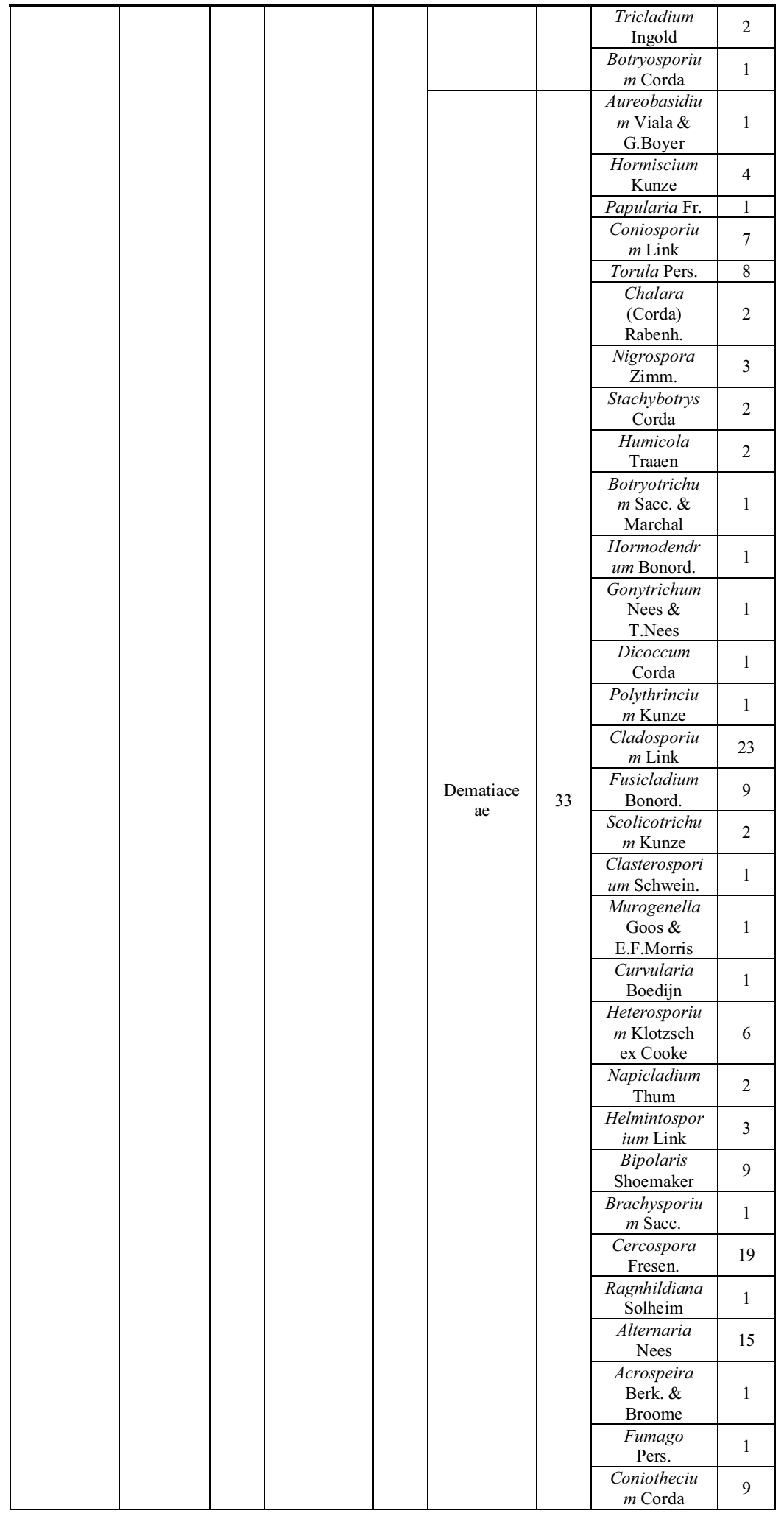




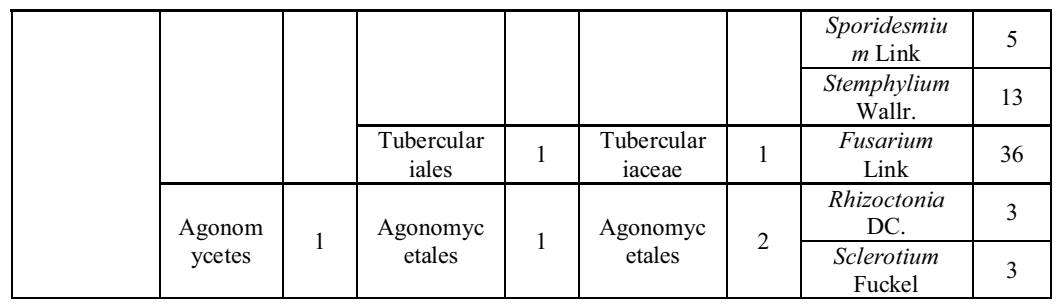

* according to Mclaughin and Spatofora [5], oomycetes are fungi-like organisms.

The representatives of the former chytridiomycetes include the phylum species Chytridiomycota, as well as Monoblepharidimycota and Blastocladiomycota. Of which Chytridiomycota includes 8 species from 4 genera, 4 families and 3 orders, Monoblepharidimycota - 2 species from 2 genera, 2 families and 1 order, Blastocladiomycota - 2 species from 2 genera, 1 family and 1 order, and in total - 12 species, 8 genera, 7 families and 5 orders. Most chytridiomycetes live in water and soil, among them there are parasites of algae, higher aquatic plants and invertebrates, as well as saprotrophs on plant and animal remains.

Representatives of true fungi were identified from zygomycetes, marsupials, basidiomycetes and the Anamorphic fungi group, the teleomorphs of which belong to Ascomycota and Basidiomycota.

Zygomycetes include 35 species from 11 genera belonging to 4 families and 1 order. Most zygomycetes are terrestrial and are saprotrophs in the soil, on plant and animal debris. Typical representatives are mucor fungi, which inhabit the soil, as well as rot-causing seeds and food products.

Marsupial mushrooms include 293 species belonging to 76 genera from 47 families, 18 orders. Based on the presence or absence of fruiting bodies and the methods of their formation, the taxonomy of ascomycetes was compiled. In the development cycle, they have two stages: perfect teleomorph and conidial - anamorphic. The marsupial fungi include economically significant families: Taphrinales and Erysiphales fungi, the causative agent of pome crops scab (Venturiaceae), Alternaria (anamorph - Pleosporales) and many other plant diseases causing diseases. Many species of ascomycetes in the anamorphic stage parasitize on various plants, causing dangerous diseases. Most of the ascomycetes, especially in the teleomorph stage, live as saprotrophs in the soil, on plant debris. Some representatives are of significant economic importance as producers of antibiotics, and growth substances (gibberellins).

Basidiomycetes include 354 species from 21 genera, 13 families, and 6 orders. The main groups of basidiomycetes include causative agents of well-known and very dangerous plant diseases: rust (Pucciniales) and smut (Ustilaginales, Urocystidales, and Tilletiales).

Imperfect mushrooms, according to the Saccardo system, include hyphae fungi, which are given in this work, include 521 species from 88 genera, 3 families, and 3 orders of magnitude in the group of deuteromycetes or Anamorphic fungi. The main families are light-colored (Moniliaceae) and dark-colored (Dematiaceae) hyphal fungi. In some deuteromycetes, conidial sporulation is absent; such species form sclerotia, or exist in the form of sterile mycelium (Agonomycetales). Among deuteromycetes, both saprotrophs that live in water, soil, plant and animal remains, and parasites that develop on higher plants, less often on animals, are known. Deuteromycetes are the cause of numerous crop diseases resulting in large crop losses. 


\section{Conclusions}

Currently, mycobiota Uzbekistan includes 816 species belonging to 122 genera, 112 families and 39 orders related to the phylum Oomycota, Chytridiomycota, Monoblepharidimycota, Blastocladiomycota, Zygomycota, Ascomycota, Basidiomycota and Anamorphic fungi (Deuteromycota).

\section{References}

1. B. K. Kalymbetov, Mikoflora of Zailiyskiy Ala-Tau, 470 (1969)

2. Sh. G. Kamilov, Kh. Kh. Nuraliev, R. K. Sattarova, A. A. Khakimov, Mycology and phytopathology, 54(5), 313-319 (2020)

3. N. Ch. Namozov, D. A. Kodirova, M. I. Usmonova, International journal of scientific \& technology research, 9(03), 5491-5493 (2020)

4. M. Urmanova, A. Kuziev, D. Burkhanova, D. Kadirova, N. Namozov, N. Shadieva, In E3S Web of Conferences, 244, 02036 (2021)

5. D. J. Mclaughin, J. W. Spatofora, The Mycota. Systematics and Evolution. Part A. N.Y.-London, 461 (2014)

6. P. M. Kirk, P. F. Cannon, D. W. Minter, J. A. Staples, Ainsworth \& Bisby’s dictionary of the fungi, 772 (CABI Europe - UK, 2008)

7. V. S. Savenko, R. A. Kulmatov, Geochemistry International, 35(11), 1028-1030 (1997)

8. B. Abdullaev, R. A. Kulmatov, A. A. Kist, Industrial Laboratory (USSR) (English translation of Zavodskaya Laboratoriya), 54(7), 710-713 (1989)

9. V. M. Emets, R. A. Kulmatov, Doklady Biological Sciences, 271(1-6), 370-372 (1983)

10. S. Islamov, N. Namozov, M. Saidova, D. Kodirova, In E3S Web of Conferences, 244, 03028 (2021) 